\title{
Creando y adaptando materiales para compartir: una experiencia con futuros maestros
}

\section{Creating and Adapting Materials to Share: An Experience with Future Teachers}

\author{
María José Fiuza Asorey*1 \\ maria.fiuza@usc.es \\ Silvia Sierra Martínez** \\ sierra@uvigo.es \\ *Universidad de Santiago de Compostela, España \\ **Universidad de Vigo, España
}

\section{Resumen:}

En el presente artículo se expone un estudio realizado durante el curso 2015-2016 en la Universidad de Santiago de Compostela. Siguiendo un proceso de investigación-acción, que contó con la participación voluntaria de 88 estudiantes de la Facultad de Formación del Profesorado, se diseñaron y/o adaptaron cuentos inclusivos en colaboración con la Organización Nacional de Ciegos Españoles (ONCE). El proceso pretende avanzar con los estudiantes en el compromiso activo con la inclusión educativa y social e iniciarles en el Aprendizaje-Servicio. En primer lugar, se reflexiona sobre los aprendizajes y el proceso seguido por los estudiantes universitarios, cuando afrontan la tarea de diseñar y adaptar cuentos actuales, generando contextos de aprendizaje compartido para niños con restos visuales, con ceguera y con visión normal. En segundo lugar se da voz a los maestros especialistas en discapacidad visual que colaboran en la formación del alumnado universitario y que realizan el seguimiento de los traba-

\begin{abstract}
:
This article presents a study carried out during the academic year 2015-2016 at the University of Santiago de Compostela. It consists of the design and/or adaptation of inclusive stories in collaboration with the Spanish National Organization of the Blind (ONCE), following an action-research process which included the voluntary participation of 88 students from the Teacher Training Faculty. This process aims at improving the students' active engagement with educational and social inclusion as well as initiating them in ServiceLearning. Firstly, we reflect on the learning and the process followed by university students as they face the task of designing and adapting current stories, creating contexts of shared learning for children with blindness as well as residual and normal vision. Secondly, a voice is given to those teachers specialized in visual impairment who collaborate in the training of university students and follow up their work, not forgetting the children, who are the ultimate recipients. The results show that the
\end{abstract}

1 Dirección para correspondencia (correspondence address):

María José Fiuza Asorey. Dpto. de Psicología Evolutiva y de la Educación. Universidad de Santiago de Compostela. Facultad de Formación del Profesorado. Avenida Ramón Ferreiro, s/n. 27002, Lugo (España). 
jos, y a los niños que son los receptores finales del trabajo realizado. Los resultados obtenidos ponen de manifiesto que la implicación en las acciones pedagógicas desarrolladas genera un mayor desarrollo de competencias profesionales y personales, a la vez que se fortalecen sinergias para la formación integral del alumnado.

\section{Palabras clave:}

Escuela Inclusiva; Aprendizaje-Servicio (ApS); Formación del Profesorado; Docencia Universitaria; Adaptación de Materiales. involvement in the implemented pedagogical actions generates a greater development of professional and personal skills while, at the same time, synergies for comprehensive training are strengthened.

\section{Key words:}

Inclusive school; Service-Learning; teacher training; university teaching; adaptation of materials.

\section{Résumé:}

Cet article présente une étude menée à bien tout au long de l'année scolaire 2015-2016 à I'Université de Saint-Jacques de Compostelle. Tout en suivant un processus de rechercheaction, soutenu par l'engagement bénévole de 88 étudiants de la Faculté de Formation des Enseignants, des contes inclusifs ont été conçus et/ou adaptés en collaboration avec I'Organisation Nationale des Aveugles Espagnols (ONCE). Ce processus a pour but de progresser au côté des étudiants sur la voie de l'engagement actif dans l'inclusion éducative et sociale et les initier ainsi à l'apprentissage-service. Une réflexion est tout d'abord présentée concernant les apprentissages et le processus suivi par les étudiants universitaires à l'heure de confronter la tâche de concevoir et adapter des contes actuels, en créant en même temps des contextes d'apprentissage partagés pour des enfants souffrant d'un handicap visuel, enfants non-voyants et enfants avec une vision normale. La voix est ensuite donnée aux enseignants d'école primaire spécialisés en handicaps visuels qui collaborent à la formation des étudiants à l'université et qui sont chargés du suivi des travaux ainsi que des enfants à qui le travail réalisé est destiné. Les résultats obtenus démontrent que l'engagement aux actions pédagogiques développées produit un essor des compétences professionnelles et personnelles, en même temps qu'un renforcement des synergies pour la formation intégrale des étudiants.

Mots-clés :

École inclusive; apprentissage-service; formation des enseignants; enseignement universitaire; adaptation de matériels.

Fecha de recepción: 16-5-2017

Fecha de aceptación: 7-9-2017

\section{Introducción}

La experiencia que se describe a continuación trata de sensibilizar al alumnado que cursa la asignatura de Dificultades de aprendizaje y trastornos del desarrollo, del grado en Educación Primaria, sobre la educación inclusiva. Para ello, se desarrolla un proyecto de Aprendizaje Servicio (ApS) consistente en la elaboración de cuentos inclusivos que son donados a la Organización Nacional de Ciegos Españoles (ONCE) para 
ser distribuidos prioritariamente en los centros educativos de Educación Infantil y Primaria de la provincia de Lugo en los que existan niños con déficits visuales y/o con ceguera. Los ejes centrales en torno a los que se desenvuelve esta experiencia son el de escuela inclusiva y el de ApS.

\section{Marco teórico}

Desde la segregación a la inclusión, pasando por la integración, el camino a recorrer en la búsqueda de una escuela y, por ende, de una sociedad más justa y equitativa es largo y no siempre libre de obstáculos. Ello se debe, fundamentalmente, al hecho de que en algunos países aún se considera a la escuela y a la educación inclusiva como una forma de prestar atención a los niños con discapacidad en el contexto de la educación general (Ainscow, 2002; y Gómez Hurtado y Ainscow, 2014) y a que las actitudes de toda la comunidad educativa, y en especial las del profesorado, son un elemento de gran relevancia para dar respuesta a la diversidad del alumnado (Garzón, Calvo y Orgaz, 2016).

Definir qué es la inclusión no es algo exento de dificultades, ya que es preciso tener presente las circunstancias locales, la cultura y la historia de cada país, pero aun así han de darse los cuatro elementos siguientes (Echeíta y Ainscow, 2011): la inclusión es un proceso, lo que quiere decir que se trata de aprender a vivir con la diferencia a la vez que se le valora como un estímulo positivo para fomentar el aprendizaje; busca la presencia, la participación y el éxito de todos los estudiantes; precisa la identificación y eliminación de barreras, es decir, de creencias y actitudes que se concretan en las culturas, las políticas y las prácticas escolares que al interactuar con las condiciones personales, sociales o culturales de determinados alumnos generan exclusión, marginación o fracaso escolar; y, finalmente, la inclusión ha de prestar especial atención a aquellos grupos de alumnos que se encuentran en riesgo de marginalización, exclusión, o fracaso escolar.

Cada vez se extiende más la idea de que es preciso lograr que todos los niños tengan un aprendizaje satisfactorio, para lo que hay que definir las políticas y estructuras educacionales, examinar los currículos, la formación pedagógica y la instrucción propiamente dicha, prestando especial atención al apoyo al aprendizaje, el bienestar y la evaluación de los estudiantes (Halinen y Jarvinen, 2008). Para alcanzar ese aprendizaje 
satisfactorio es preciso recordar el concepto de "barreras al aprendizaje y la participación" con el que, de manera genérica, Booth y Ainscow (2000) definen los obstáculos que los alumnos encuentran para aprender y participar. No es excepcional que el profesorado considere que la diversidad constituye una de esas barreras al entenderse las diferencias como un importante hándicap y no como una situación que aporta beneficios a todos (Fernández, Fiuza y Zabalza, 2013). Como señala Echeita (2007), para disminuir la brecha existente entre los valores declarados sobre la educación inclusiva y la realidad de las aulas se requiere reestructurar algunas concepciones arraigadas en las personas que constituyen el contexto educativo y entre las que se encuentra el profesorado. En este sentido, destacan las palabras de León y Arjona (2011) al subrayar que para alcanzar una educación para todos es condición básica que el profesorado esté suficientemente cualificado, de tal forma que sea capaz de convertirse en profesionales más reflexivos y críticos.

Independientemente de las características y necesidades presentadas, los centros educativos han de dar respuesta a su alumnado. Siguiendo a Ainscow (2002), la meta de conseguir una educación inclusiva pasa por eliminar la exclusión social derivada de actitudes y respuestas a la diversidad de raza, clase social, origen étnico, religión, género y logros, como así también de capacidades; es decir, requiere ser capaz de pensar que todos los niños y niñas pueden y deben aprender (Darling-Hammond, 2001). Para lograr este objetivo es necesario que se produzcan cambios dentro del contexto de la educación mediante acciones dirigidas a fomentar una cultura de escuela desarrollada en torno a la comunidad, con estructuras organizativas centradas entre otros elementos en la formación de equipos colaborativos, procesos de solución de problemas y redefinición del currículum, a la vez que las clases inclusivas abran sus puertas a la enseñanza individualizada, la co-enseñanza, la enseñanza cooperativa, las adaptaciones curriculares, o la acomodación de la instrucción y evaluación de su alumnado (León y Arjona, 2011). En definitiva, se trata de transformar las pedagogías de la escuela actual hasta alcanzar respuestas a las necesidades derivadas de la diversidad existente en las instituciones educativas (Parajes y López, 2012). Y si fuese posible simplificar el entramado de la educación inclusiva habría que decir que las piezas clave son la formación y la colaboración (Moriña y Parrilla, 2006).

Las respuestas de calidad a la diversidad han de partir de planteamien- 
tos equitativos y con un fuerte carácter preventivo y global, dejando atrás los enfoques asistenciales, compensatorios o focalizados y lograr su consolidación exige la determinación y la convicción de los profesionales implicados (Muntaner, Roselló y De la Iglesia, 2016). Como reflexionan Escudero y Martínez (2011), la educación inclusiva pertenece al dominio de utopías realistas que, por complejas, difíciles y lejanas que estén, deben inspirar políticas, culturas y prácticas, con un enfoque no inspirado en opciones caritativas y particulares sino en imperativos morales y de justicia social. La fuerza y el empuje de la educación inclusiva la convierten en un movimiento mundial cuya bandera es la lucha contra la selección y la competitividad, que propone políticas no segregadoras, cuya máxima aspiración es la inclusión de todos los ciudadanos más allá del contexto educativo (Arnaiz, 2012).

El cambio que la adaptación al Espacio Europeo de Educación Superior (EEES) ha generado en las universidades españolas Ileva implícita la incorporación cada vez más frecuente de las denominadas metodologías activas, junto con la aplicación de un sistema de evaluación continuo o formativo (Palomares, 2011). En ese cambio de metodología desde la clase magistral a un aula en la que se busca la participación activa del alumnado se destaca el papel concedido al aprendizaje significativo, activo y experiencial en contextos más o menos próximos. El ApS es un buen ejemplo de ello.

En palabras de Puig y Palos, el ApS es una propuesta educativa que combina procesos de aprendizaje y de servicio a la comunidad en un solo proyecto bien articulado en el que los participantes se forman al trabajar sobre necesidades reales del entorno con el objetivo de mejorarlo (2006, p. 61). Se traza así un puente entre lo académico y lo social (GilGómez, Moliner-García, Chiva-Bartoll y García-López, 2016), en el que se vinculan los contenidos curriculares con el servicio a la comunidad. Las características definitorias del ApS son la relación directa y significativa con la realidad, la participación activa en las diferentes fases del proyecto, la reflexión, la interdisciplinariedad y resolución de problemas, la cooperación y la adquisición de valores (Puig y Palos, 2006).

Aunar en un mismo proyecto las prácticas inclusivas con el ApS es una buena forma de partir de hojas de ruta con muchos puntos en Común para dar un paso más hacia la inclusión y la solidaridad (Abal de Hevia, 2016). En ambas formas se perfila la educación como un proceso activo en la búsqueda de la prosocialidad, creando sinergias y enrique- 
ciendo el Ilamado "capital social" del centro escolar en las relaciones interpersonales o sociales generadas (Mendía, 2012).

En palabras de Agrafojo, García y Jato (2017, p.26), "el auge del ApS en la Educación Superior está ligado a una activa relación de la universidad con la sociedad civil", lo que se materializa en el volumen de experiencias de ApS que en los últimos años se están realizando en España como son, entre otros, los trabajos de Cuevas, Chiva y Francisco (2016); García y Sánchez (2017); y Zayas y Martínez-Usarralde (2017).

La experiencia que se detalla a continuación conjuga inclusión y ApS, prestando atención a los componentes que configuran el ApS y que le definen y diferencian del voluntariado o del aprendizaje basado en la comunidad: el protagonismo de la voz del alumnado, la atención a una necesidad real existente en la comunidad, la conexión con los contenidos curriculares de aprendizaje, la ejecución de un proyecto de servicio y, finalmente, la reflexión que conduce a la evaluación continuada de las fases del proyecto y que garantiza la interiorización de los objetivos de aprendizaje curricular (Martínez-Odría, 2007). Se trata de un proyecto en el que alumnado de la asignatura de Dificultades de aprendizaje y trastornos del desarrollo (obligatoria en el segundo semestre del primer curso para la obtención del título de Graduado o Graduada en Maestro de Educación Primaria en la Facultad de Formación del Profesorado del Campus de Lugo, Universidad de Santiago de Compostela) en colaboración con la Organización Nacional de Ciegos Españoles (ONCE, delegación de Lugo) elabora cuentos inclusivos.

En la formación del estudiantado del grado de primaria en la Universidad de Santiago de Compostela no se contempla de forma clara y directa el abordaje de la diversidad con la mirada de la educación inclusiva. Aun considerando que muy probablemente exista profesorado comprometido con esta temática, el alumnado corre el riesgo de finalizar sus estudios sin ahondar suficientemente en el concepto y el sentir de la escuela inclusiva. Surge así la iniciativa de crear cuentos y maquetas inclusivos, lo que significa que son materiales para compartir niños con visión normal, niños con diferentes grados de discapacidad visual y niños con ceguera.

Al menos en los ámbitos más ligados a la docencia, se reconoce la labor que tanto el profesorado de educación infantil como el de primaria, y especialmente el primero de ellos, dedica a la elaboración y adaptación de materiales que den respuesta a las necesidades de su alumnado. 
Los materiales educativos son potentes herramientas que enriquecen y concretan el aprendizaje y no ha lugar a discusión alguna sobre sus efectos positivos en la eficacia de la educación. Dentro de este amplio abanico de tareas dada la diversidad de necesidades del alumnado receptor, y seguramente no suficientemente reconocidas por parte de la administración pública, hay que hacer una especial mención a los materiales pensados para el alumnado con necesidades educativas especiales. Como también es preciso destacar el esfuerzo que supone no solo la elaboración de materiales sino la adquisición de la formación específica imprescindible para que todo ese trabajo se plasme en materiales que cumplan el objetivo para el que han sido pensados. Sin embargo, no siempre el resultado refleja una comprensión amplia de la educación inclusiva ya que tienden a centrarse bien en el aula en general, bien en las necesidades especiales. Se crea así, sin conciencia de ello, una barrera más a la plena inclusión.

\section{Objetivos}

En el marco de la investigación-acción en la que se desarrolla este trabajo el objetivo general es profundizar en prácticas inclusivas a través de un proyecto de ApS.

Los objetivos específicos son los siguientes:

- Erradicar creencias erróneas sobre la inclusión.

- Iniciarse en el ApS.

- Profundizar en las necesidades educativas del alumnado con diversos grados de pérdida visual dando respuesta a dichas necesidades.

- Desarrollar materiales adaptados para alumnado que presenta una pérdida de visión o ceguera.

- Dar voz a los participantes en el proyecto a partir de sus reflexiones sobre el mismo.

- Obtener información que permita mejorar la propuesta de ApS en cursos posteriores. 


\section{Metodología}

\section{Participantes}

En este estudio han participado un total de 88 alumnos matriculados en la asignatura de Dificultades de aprendizaje y trastornos del desarrollo del Grado de Educación Primaria, de los que 62 eran mujeres y 26 varones; los dos maestros itinerantes de la provincia de Lugo especialistas en discapacidad visual (un varón y una mujer); y, por último, se eligieron al azar tres niñas con visión normal (una tiene 6 años y dos 7 años), tres con restos visuales (un niño de 5 años y dos niñas con 5 y 6 años) y tres con ceguera (dos niñas de 6 años y una de 7), escolarizados en diferentes centros educativos de dicha provincia.

En cuanto al alumnado de la Facultad de Formación del Profesorado de Lugo, la elaboración de los cuentos constituye el trabajo obligatorio de la asignatura, de 6 créditos ECTS, y se realiza en la hora y media semanal que se dispone para las sesiones de grupo mediano (o grupo interactivo, constituido por un tercio del total del alumnado). Aunque es el trabajo obligatorio de la asignatura se ofrece al alumnado la posibilidad de no participar en el mismo, sustituyéndose por un trabajo individual o en pequeño grupo (si existen más personas que también eligen esta segunda opción), de corte más tradicional. Desde que se inicia esta experiencia, ningún alumno o alumna sin exención de docencia ha optado por esta posibilidad.

El convenio de colaboración con la ONCE se renueva cada curso escolar y gracias a él el alumnado recibe tres sesiones de formación sobre cómo elaborar los cuentos, atendiendo a las necesidades de aquellos a quienes va dirigido: niños con ceguera, con restos visuales y niños con visión normal.

Una vez finalizado el período formativo, el asesoramiento ante posibles dudas se mantiene mediante el correo electrónico y/o reuniones presenciales una vez por semana en las dependencias de la agencia provincial de la ONCE, lo que también permite el seguimiento del trabajo, la introducción de mejoras, etc... En general, las consultas suelen centrarse en dudas sobre el código Braille, la utilización de la máquina Perkins y en la elección de los materiales más apropiados para trabajar las diferentes texturas. 

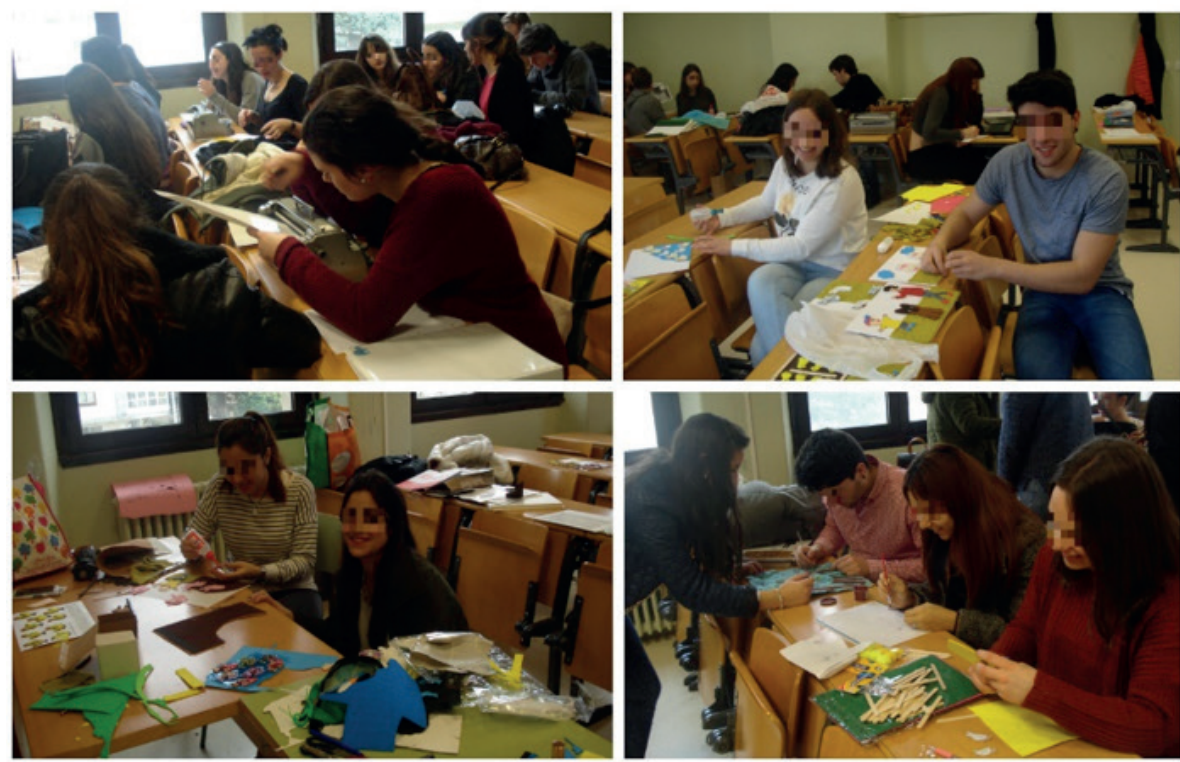

Imagen 1. Proceso de elaboración de material inclusivo adaptado por parte del alumnado universitario.

\section{Instrumentos}

Se han utilizado diferentes técnicas de recogida y producción de datos según el grupo al que estaban dirigidas y su finalidad. En relación a la voz del alumnado universitario, una vez finalizados los cuentos, se procede a realizar una jornada de reflexión y análisis de la experiencia vivida. La sesión de reflexión consta de dos partes: en un primer momento se escuchan sus aportaciones y, a continuación se les incita a que respondan a varias cuestiones de interés: "cómo alumno/a, qué aspectos positivos destacarías de esta experiencia?", "como futuro maestro/a, ¿qué aspectos positivos destacarías de la experiencia?", "¿qué propuestas de mejora propondrías en la experiencia de colaboración con la ONCE?" y "tras realizar esta experiencia de ApS, ipiensas en incluir experiencias similares en tu docencia como maestro/a?". Se analizan las respuestas emitidas tanto sobre los aprendizajes realizados como las vivencias generadas a lo largo del proceso.

Los dos profesores especialistas en discapacidad visual responden vía on-line a un cuestionario con dos preguntas abiertas "¿qué crees que aporta este proyecto en las aulas en las que se utiliza el material elaborado por 
el alumnado de la Facultad de Formación del Profesorado?" y "¿en qué aspectos consideras que puede mejorarse?". Y, para finalizar, se recogen las voces de los destinatarios finales de los materiales elaborados. Para esta tarea se ha contado con la ayuda de los profesores especialistas que, aprovechando su visita a los centros en lo que hay escolarizados niños y niñas con problemas de visión, aprovechan su estancia para entrevistarles, a ellos y a sus compañeros con visión normal. Las preguntas a las que han dado voz son las siguientes: "¿qué te parece que todos podáis leer el mismo cuento?", "¿qué es lo que más te ha gustado de estos cuentos?", "iqué es lo que menos te ha gustado?" y "¿qué otras cosas te gustaría compartir?".

\section{Análisis de datos}

La información recogida entre el alumnado universitario, profesorado especialista y alumnado de educación infantil y educación primaria fue sometida a un primer análisis de contenido en base a grandes categorías temáticas de análisis. El análisis, de acuerdo con las cuestiones de investigación, incluyó las siguientes categorías: visión del alumnado universitario, visión del profesorado especialista en discapacidad sensorial y percepción del alumnado destinatario de los materiales adaptados. Estas categorías son las que se han respetado y se mantienen en el análisis de datos que se incluye en el siguiente apartado.

\section{Resultados}

A continuación se exponen los resultados obtenidos de los tres colectivos participantes en la experiencia.

\section{Visión de alumnado universitario}

El análisis de los resultados cualitativos del alumnado universitario tras la jornada de reflexión saca a la luz el empoderamiento del mismo en tres aspectos claves de su formación: el aprendizaje, la cohesión que genera el trabajo colaborativo y la formación en valores. Por lo que respecta a los aprendizajes, van más allá de lo meramente académico, abarcando lo social y prestando especial atención a la inclusión. Las siguientes palabras son el sentir de futuros docentes de educación primaria: 
"Aprendimos mucho más de las deficiencias visuales que si lo hubiésemos estudiado" (Mujer, 20 años).

"Aprendimos cómo se empieza a enseñar braille a los niños y saber cómo adaptar un libro a sus necesidades" (Mujer, 18 años).

"Con esta experiencia pude adquirir conocimientos que serán de utilidad para dar respuesta a las necesidades de mis alumnos y poder acercarme a una exigencia curricular como es la educación en valores y la atención a la diversidad" (Varón, 20 años).

"Me parece muy importante conocer cómo se debe trabajar con estos niños en cuanto a materiales didácticos: qué materiales usar, cuántos, los colores..." (Mujer, 18 años).

El aprendizaje deja de ser considerado como la adquisición de conocimientos de forma individual para integrarse en un proceso de participación social. La competitividad desaparece para dar lugar a la búsqueda de metas comunes, generándose una gran cohesión intragrupo pero también intergrupos.

"Ha sido un trabajo colaborativo y cooperativo que ha permitido una mejor interacción con los compañeros, Ilegar a conclusiones conjuntas..." (Varón, 20 años).

"Destacaría el buen rollo, el respeto y el compañerismo que se da en los grupos de trabajo, ayudándonos unos a otros" (Varón, 20 años).

"Destacaría como aspectos positivos el trabajo colaborativo y cooperativo, este tipo de actividades permiten una mejor interacción con los compañeros y permiten conocerse mejor" (Mujer, 20 años).

El alumnado es consciente de que la experiencia les enriquece y les hace crecer. Les posibilita el acceso a la reflexión y la conciencia social, construyendo experiencias positivas, estados psicológicos que mejoran su competencia psicosocial y autonomía personal.

"Tras esta experiencia, me gustaría ser voluntaria para el siguiente curso académico y ampliar los nuevos conocimientos y experiencias en estos campos, conocer otras realidades" (Mujer, 26 años).

"Es un proyecto divertido de concienciación" (Mujer, 19 años).

"Considero que en la realización de este cuento todo fueron factores positivos: el poder realizar un trabajo que no solo esté destinado a ser calificado 
y sobre todo que tiene una utilidad, el saber que tu esfuerzo y dedicación tendrán su recompensa sirviéndole de gran ayuda a niños que quieren disfrutar de un cuento..." (Mujer, 18 años).

\section{Visión del profesorado especialista en discapacidad sensorial}

Por otro lado, los dos profesores especialistas en discapacidad sensorial optaron por enviar sus respuestas de forma conjunta, tras un tiempo de diálogo y reflexión. Consideran que la experiencia tiene un marcado carácter inclusivo, al ser válidos los cuentos para todos los niños, destacando su atractivo para el alumnado con visión normal y la aportación que supone para el profesorado.

"...suelen sentir curiosidad por tocar el braille y quieren saber cómo su compañero/a invidente puede leer utilizando las manos. La mayoría del alumnado no sólo lee el libro en tinta sino que, también explora táctilmente los elementos en relieve y los puntos braille".

"Al profesorado, le aporta una nueva visión acerca de la posibilidad de utilizar un único recurso válido para todo el alumnado, independientemente de las características que presenten. Constituye un recurso que les sirve de modelo para la creación de nuevos elementos didácticos (fichas adaptadas muy variadas)".

Como seguramente no podría ser de otro modo hay aspectos que requieren ser mejorados. Los maestros especialistas destacan los errores de transcripción al código braille y el material específico utilizado para la fijación de los diferentes elementos que componen el cuento.

"Habría que intentar no tener erratas porque se trata de edades donde los niños no suelen deducir la palabra en función del contexto, sobre todo en educación infantil".

"Hay que mejorar la fijación de los elementos en relieve dado que algunos se despegan después de un uso relativamente corto".

\section{Visión del alumnado destinatario de los materiales adaptados}

Por último, el alumnado de educación infantil y educación primaria destinatario de los materiales adaptados, manifiesta estar muy satisfecho 
con la experiencia de compartir cuentos educativos. Se exponen a continuación las respuestas de los participantes en la experiencia en las que se plasma el acercamiento que esta supone al mundo de los niños con discapacidad y, a su vez, ayuda a visibilizar la realidad del alumnado con ceguera o restos visuales.

Profesor especialista: ¿Qué te parece que todos podáis leer el mismo cuento?

Alumna (6 años) con visión normal: "Muy bien. Yo no sabía que había cuentos para niños ciegos y me gusta mucho que todos los podamos leer".

Alumna (7 años) con visión normal: "Muy bien. A mí me gusta poder leer estos libros aunque no entiendo los puntitos".

Alumna (7 años) con resto de visión: "Bien porque yo tengo que usar la lupa con los otros".

Alumna (5 años) con resto de visión: "Bien, así valen todos para todos".

Alumna (6 años) con ceguera total: "Bien, la profe les dice a los demás que así saben cuáles son mis dificultades".

Alumna (7 años) con ceguera total: "La mejor idea del mundo".

En lo referente al diseño de los cuentos, el alumnado de educación infantil y de educación primaria destaca tanto la estética de las ilustraciones y los dibujos elaborados por el alumnado universitario como la legibilidad de los mismos debido al tamaño y al relieve.

Profesor especialista: ¿Qué es lo que más te ha gustado de estos cuentos?.

Alumna (7 años) con visión normal: "Todas las cosas que tienen y que son cuentos para tocar y mirar".

Alumna (7 años) con visión normal: "Lo que más, las ilustraciones. Son muy bonitos y llaman mucho la atención".

Alumna (6 años) con resto de visión: "A mí, las letras grandes porque se lee mejor".

Alumna (6 años) con resto de visión: "Son chulísimos, los dibujos los veo muy bien".

Alumna (6 años) con ceguera total: "Tienen braille y dibujos que se pueden tocar".

Alumna (7 años) con ceguera total: "Los dibujos que tienen". 

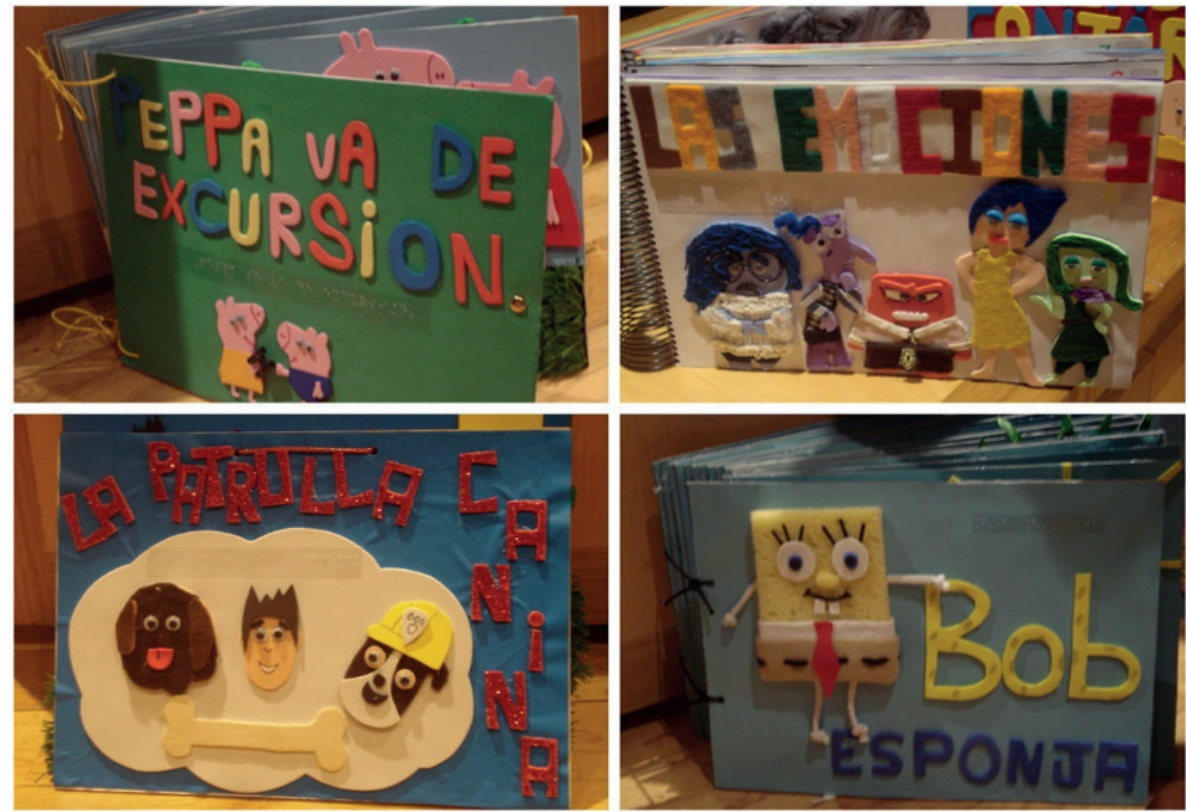

Imagen 2. Muestra de los cuentos educativos adaptados.

Aunque el alumnado valora positivamente la iniciativa también aporta recomendaciones y sugerencias de cara a futuras experiencias de adaptación de cuentos infantiles como, por ejemplo, el tamaño de las letras o la fragilidad del material.

Profesor especialista: “¿Qué es lo que menos te ha gustado?"

Alumna (6 años) con visión normal: "Que no se pueden Ilevar para casa" (niña, 6 años).

Alumna (6 años) con visión normal: "Que son muy grandes. Que hay que andar con mucho cuidado porque son muy frágiles".

Alumna (6 años) con resto de visión: "Las letras aún son pequeñas".

Alumna (5 años) con resto de visión: "No sé, me gustó todo".

Alumna (7 años) con ceguera total: "Hay palabras mal escritas, a veces".

Alumna (6 años) con ceguera total: "Que se suelten los dibujos".

Asimismo el alumnado de educación infantil y educación primaria también propone la adaptación de otros materiales de uso habitual dentro del aula como el calendario escolar, los juegos de ordenador, las imágenes de los libros de texto o los carteles educativos colgados en el 
aula. En la misma línea, proponen compartir con el resto de sus compañeros la maquina Perkins o el material ya adaptado que la ONCE pone a disposición del alumnado dentro del aula.

Profesor especialista: ¿Qué otras cosas te gustaría compartir?

Alumna (6 años) con visión normal: "La máquina de escribir".

Alumna (7 años) con visión normal: "Las cosas que le trae la chica de la ONCE".

Alumna (6 años) con resto de visión: "Los juguetes y puzles".

Alumna (5 años) con resto de visión: "El calendario, mejor más grande".

Alumna (7 años) con ceguera total: "Todas las imágenes de los libros de texto, el mío trae muy poquitas".

Alumna (7 años) con ceguera total: "Los carteles que la profe dice que están en la pared, esos, yo no los veo, claro".

En definitiva, no cabe duda de que el profesorado es sensible a las necesidades de su alumnado y de que, por tanto, busca la flexibilidad en el proceso de elaboración y/o diseño de materiales diversos, de tal modo que puedan tener múltiples utilidades en contextos de aprendizaje compartidos. Pero también es necesario hacer mención a que hay que ir más allá de las buenas intenciones y que no siempre lo que aparenta ser inclusivo lo es en realidad. Se hace imprescindible confesar, humildemente, que el primer curso académico (2012-2013) en que se apostó por sensibilizar al alumnado con la inclusión y para ello se propuso la realización de cuentos para niños con ceguera que serían donados a la ONCE, el resultado no fue inclusivo. Crear cuentos para niños con ceguera no es inclusión. Crear cuentos solo para algunos niños, no es inclusivo. Lo inclusivo es crear cuentos para todos, cuentos para compartir, se tenga una visión normal, restos visuales o ceguera. En ello estamos.

Al elaborar cuentos verdaderamente inclusivos y no solo para niños con ceguera o con restos visuales, es necesario escribirlos en código braille pero también en lengua castellana o lengua gallega, según elija el alumnado y siguiendo unos parámetros fijados previamente para poder ser válidos para el alumnado que presenta una pérdida de visión leve o moderada. Se trabaja el color por el gran poder de atracción que presenta para los niños con visión normal y/o con restos visuales, y se presta especial atención a las texturas, muy importantes para los niños con ceguera e igualmente estimulantes para los demás niños. Los cuentos se 
realizan en la opción que el alumnado elige de entre las dos siguientes: por un lado, se puede realizar una adaptación de algún personaje famoso entre los niños como "la patrulla canina", "Peppa Pig" o "Pocolló", por citar algunos ejemplos, y sintetizar el contenido o idear uno nuevo para desarrollar conceptos abstractos como la amistad, el compañerismo, etc.; por el otro, existe la posibilidad de, partiendo de algunos de estos personajes o ideando otros nuevos, realizar un relato en el que se trabajan conceptos básicos de corte más académico (tamaños, formas..) que están especialmente dirigidos a los niños con sordoceguera.

Si bien fue en el curso 2013-2014 cuando se realizó el primer proyecto de colaboración entre la Facultad de Formación del Profesorado y la ONCE, el presente trabajo, de forma especial, centra la atención en torno al análisis de las voces de todos los participantes en el proyecto durante el curso 2015-2016. Atendiendo a las necesidades presentadas por los dos profesores itinerantes, se realizaron cuentos educativos adaptados como respuesta a su solicitud de elaboración de material adaptado para niños de educación primaria con sordoceguera desde el Centro de Recursos de la ONCE de Pontevedra. En primer lugar se escucha la voz del alumnado universitario $y$, acto seguido, se dedica un espacio y un tiempo al profesorado itinerante en la provincia de Lugo especialista en discapacidad visual y encargado de la distribución de los materiales. La labor de dichos especialistas es doble: por un lado, forman a los futuros docentes sobre las necesidades de elaboración de los materiales para que cumplan su misión inclusiva y, por el otro, sirve de puente entre ellos y los destinatarios finales del servicio. Finalmente, son los niños y niñas receptores del servicio los que expresan el significado concedido al material recibido y narran sus vivencias al respecto.

\section{Discusión}

La innovación educativa lleva implícita cambios de calado en la forma de pensar y planificar la enseñanza, realizando modificaciones dirigidas a favorecer el aprendizaje del alumnado, desarrollando metodologías activas y participativas que amplían el escenario del aprendizaje posibilitando su funcionalidad y la construcción de esquemas mentales resultantes de la toma de contacto con la realidad (Cámara y Pegalajar, 2014). Ello supone adherirse a un modelo de universidad que busca la calidad 
pero que se orienta a la formación de titulados que actúan desde perspectivas orientadas al logro del bien común y de una sociedad más justa y democrática (Martínez, 2010). En esa línea se desarrolla el proyecto de colaboración con la ONCE: nuevas formas y nuevos modos de ahondar en el aprendizaje buscando una sociedad más equitativa.

La realización de cuentos inclusivos permite al alumnado universitario una mayor reflexión sobre la discapacidad en general y la discapacidad visual en particular, desde la mirada de la escuela inclusiva. Profundizar en la inclusión significa aceptar la realidad y las características de todo el alumnado desde una mirada positiva (Muntaner, Roselló y De la Iglesia, 2016). Entender que los materiales diseñados han de servir para todos y no solo para una parte del alumnado es un paso de gigante que el alumnado ha dado al participar en este proyecto, como así lo indican las reflexiones del profesorado especialista.

La posibilidad de realizar aprendizajes Ilevándolos a la práctica consolida las adquisiciones realizadas y motiva al alumnado a seguir aprendiendo (recuérdense las palabras de una alumna, citadas anteriormente, cuando dice "Aprendimos mucho más de las deficiencias visuales que si lo hubiésemos estudiado"). El alumnado reclama un cambio en la forma de aprender, y el ApS es una buena manera de acercarnos a ello. Como señalan Puig, Gijón, Martín y Rubio (2011), aprender haciendo es uno de los principios básicos de la pedagogía moderna que permite introducir el concepto de aprendizaje implícito en las actividades de aprendizaje- servicio.

Desde el socioconstructivismo, el aprendizaje ya no es únicamente la adquisición de conocimientos de un individuo porque adquiere un marcado carácter social, en el que la meta a conseguir es el desarrollo de personas reflexivas y autónomas y la responsabilidad es individual pero también grupal (Labrador-Piquer y Andreu-Andrés, 2014). El emprender trabajos académicos realizados en grupo, en el que todos colaboran para obtener una meta compartida, facilita la cohesión entre el alumnado. Pero si esa tarea tiene un trasfondo de mejora de la sociedad, hace reflexionar sobre el mundo en que vivimos y el mundo que en queremos vivir, logra que no solo se trate de hacer lo mejor posible la tarea encomendada sino que se colabore con los demás, en el equipo del que se forma parte y con el resto de los equipos, aportando ideas de mejora, prestando materiales, ayudando en lo que sea preciso...Porque se trata de formar en valores, algo que primero "sucede" y que cuando se está 
a punto de finalizar el proceso se convierte en una forma de sentir, de vivenciar el aprendizaje: "destacaría el buen rollo, el respeto y el compañerismo que se da en los grupos de trabajo, ayudándonos unos a otros".

Las aportaciones de los niños a los que están destinados los cuentos inclusivos ponen de manifiesto que el objetivo de crear "cuentos para compartir" es una realidad. Los niños, tengan el grado de visión que tengan, comparten cuentos diseñados que facilitan el acercamiento a la discapacidad y las diferencias: "yo no sabía que había cuentos para niños ciegos y me gusta mucho que todos los podamos leer", dice una niña con visión normal, "... así los demás saben cómo leo", afirma una niña con ceguera. "Son muy bonitos y llaman mucho la atención", manifiesta una niña con visión normal, porque son estimulantes y llenos de atractivo para todos. Este acercamiento al mundo de la discapacidad visual hace que los niños quieran compartir más cosas y de ahí sus peticiones al respecto: los niños con visión normal quisieran jugar con las cosas que traen los maestros especialistas para los niños con resto de visión o con ceguera, los niños con resto visual quisieran que se les adaptasen más materiales a sus necesidades específicas ya que ahora no tienen acceso a ellos dado el tamaño de las letras e imágenes y los niños con ceguera también desean poder acceder a más materiales de uso común en el aula. Esta experiencia ha despertado su interés por compartir, por crear espacios comunes para todos.

\section{Conclusión}

Las buenas prácticas inclusivas proporcionan programas de alta calidad educativa para todos y se centran en la presencia, la participación y los logros de todo el alumnado y estos avances significan cambios importantes en el sistema educativo y en el desarrollo de escuelas inclusivas (Muntaner, Roselló y De la Iglesia, 2016). Si esta se conjuga con una experiencia de ApS se está realizando una importante aportación a nuevos modos de encarar el proceso de enseñanza-aprendizaje en la universidad, generando cambios en la forma en que se enseña a los futuros maestros y en cómo estos aprenden, pero también en el estilo docente que en el futuro desarrollarán en sus aulas. Promover conductas prosociales en el alumnado universitario, adquiriendo un compromiso con una sociedad más justa y equitativa, es una buena forma de abrir la 
universidad al mundo, a la realidad, y de ofrecer una fuerte resistencia y eliminar las barreras de quienes muestran reticencias hacia el cambio que supone la implementación de políticas y prácticas que repercuten en la mejora de la calidad y la equidad en la educación para todo el alumnado.

En ese largo camino que todavía queda por recorrer en la búsqueda de una escuela y de una sociedad más justa y equitativa es fundamental acometer proyectos que con un marcado carácter innovador vayan derribando barreras a la inclusión. Es necesario que hablar de diversidad en la escuela sea hablar de una realidad en la que cualquier persona está participando en la escuela de su comunidad (Parrilla, 2002). No cabe duda que hay mucho que avanzar pero el camino se hace andando y levantándose ante las piedras que puedan hacernos tropezar e incluso caer. Quienes participan en este proyecto tienen claro que, al menos, hay dos cuestiones que necesitan ser mejoradas: en primer lugar, es preciso mejorar la calidad del material escolar utilizado para adaptar y crear cuentos con el fin de ofrecer, en la medida de lo posible, recursos educativos perennes que resistan el uso que hace de los mismos el alumnado de educación infantil y educación primaria; en segundo lugar, se debe sensibilizar al alumnado universitario sobre la formación y el cuidado que exige el uso de máquinas específicas como la máquina Perkins.

Desarrollar acciones como esta experiencia de elaboración de materiales inclusivos en colaboración con la ONCE se muestra como una buena contribución para enriquecer la imagen que la comunidad tiene sobre la universidad, dándole una mayor visibilidad al acercarla a la sociedad y posibilitar su consideración como un espacio cualificado que colabora en la resolución de retos a los que la sociedad se enfrenta.

\section{Referencias bibliográficas}

Abal de Hevia, I. (2016). Aprendizaje servicio solidario: una propuesta pedagógica innovadora. RIDAS, Revista Iberoamericana de Aprendizaje y Servicio, 2, 3-32.

Ainscow, M. (2002). Haciendo que las escuelas sean más inclusivas: lecciones a partir del análisis de la investigación internacional. Revista Educación Inclusiva, 5 (1), 39-49.

Ainscow, M.; Booth, T. y Dyson, A. (2006). Improving schools, developing inclusion Londres: Routledge.

Agrafojo, J.; García, B.; Jato, E. (2017). Aprendizaje servicio e innovación educativa en 
Creando y adaptando materiales para compartir: una experiencia con futuros maestros María José Fiuza Asorey y Silvia Sierra Martínez

la Universidad de Santiago de Compostela: estrategia para su institucionalización. RIDAS, Revista Iberoamericana de Aprendizaje Servicio, 3, 23-34.

Arnaiz, P. (2012). Escuelas eficaces e inclusivas: cómo favorecer su desarrollo. Educatio Siglo XXI, 30 (1), 25-44.

Booth, T., Ainscow, M., (2000). Index for Inclusion: developing learning and participation in schools. Bristol: Centre for Studies on Inclusive Education (CSIE).

Cámara, A. M. y Pegalajar, M. C. (2014). Percepciones de futuros docentes sobre la metodología de Aprendizaje-Servicio. En II Congreso Virtual Internacional sobre Innovación Pedagógica y Praxis Educativa. INNOVAGOGíA. Sevilla, 26-28 marzo, 617-626.

Cuevas Goterris, E., Chiva Bartoll, Ò., y Francisco Amat, A. (2016). Análisis sobre la personalidad eficaz en alumnado de didáctica de la educación física a través de aprendizaje-servicio. Revista Inclusiones, 3(2), pp. 152-169.

Darling-Hammond, L. (2001). El derecho de aprender. Crear buenas escuelas para todos. Barcelona: Ariel.

Echeita, G. (2007). Del dicho al hecho hay gran trecho. Revista Latinoamericana de Educación Inclusiva, 1 (1), 29-36.

Echeita, G. y Ainscow, M. (2011). La educación inclusiva como derecho. Marco de referencia y pautas de acción para el desarrollo de una revolución pendiente. Tejuelo, 12 26-46.

Escudero, J. M. y Martínez, B. (2011). Educación inclusiva y cambio escolar. Revista Iberoamericana de Educación, 55, 85-105.

Fernández, C. J., Fiuza, M. y Zabalza, A. (2013). A propósito de cómo analizar las barreras a la inclusión desde la comunidad educativa. Revista de Investigación en Educación, 11 (3), 172-191.

Folgueiras, P., Luna, E. y Puig, G. (2013). Aprendizaje y servicio: estudio del grado de satisfacción de estudiantes universitarios. Revista de Educación, 362. Disponible en http://www.mecd.gob.es/revista-de-educacion/numeros-revista-educacion/numerosanteriores/2013/re362/re362_06.html

Furco, A. (2004). El impacto educacional del aprendizaje servicio ¿Qué sabemos a partir de la investigación? University of California-Berkeley. Disponible en: http://roserbatlle.files. wordpress.com/2009/02/resultatsinvestigacio-aps-furco-modo-de-compatibilidad.pdf

García García M. y Sánchez Calleja, L. (2017). El aprendizaje servicio y el desarrollo de las competencias emocionales en la formación inicial del profesorado. Contextos Educativos. Revista de Educación, (20), 127-145.

Garzón, P., Calvo, M. I. y Orgaz, M. B. (2016). Inclusión educativa. Actitudes y estrategias del profesorado. Revista Española de Discapacidad, 4 (2), 25-45.

Gil-Gómez, J., Moliner-García, O., Chiva-Bartoll, O. y García-López, R. (2016). Una experiencia de aprendizaje-servicio en futuros docentes: desarrollo de la competencia social y ciudadana. Revista Complutense de Educación 27 (1), 53-73. Disponible en: http://dx.doi.org/10.5209/rev_RCED.2016.v27.n1.45071

Halinen, I, Jarvinen, R. (2008). En pos de la educación inclusiva: el caso de Finlandia. Perspectivas, XXXVIII (1), 97-127.

Gómez Hurtado, U. y Ainscow, M. (2014). Hacia una escuela para todos: liderazgo y colaboración. Revista Investigación en la Escuela, 82, 19-30. 
Labrador-Piquer, M. J. y Andreu-Andrés, M. A. (2014). Investigación-acción para conseguir grupos colaborativos eficaces. Educatio Siglo XXI, 32 (3), 75-98. Disponible en: http://dx.doi.org/10.6018/j/210991

León, M. J. y Arjona, Y. (2011). Pasos hacia la inclusión escolar en los centros de educación secundaria obligatoria. Innovación Educativa, 21, 201-221.

López, M., Echeita, G. y Martín, E. (2010). Dilemas en los procesos de inclusión: explorando instrumentos para una comprensión de las concepciones educativas del profesorado. Revista Latinoamericana de Educación Inclusiva, 4 (2). Disponible en: http://www.rinace.net/rlei/numeros/vol4-num2/art8_htm.html

Martínez, M. (2010). Aprendizaje servicio y construcción de una ciudadanía activa en la universidad: la dimensión social y cívica de los aprendizajes académicos. En Miquel Martínez (ed.), Aprendizaje servicio y responsabilidad social de las universidades (11-26). Barcelona: Octaedro.

Martínez-Odría, A. (2007). Service-learning o aprendizaje-servicio. La apertura de la escuela a la comunidad local como propuesta de educación para la ciudadanía. Bordón, 59 (4), 627-640.

Mendía, R. (2012). El Aprendizaje-Servicio como una estrategia inclusiva para superar las barreras al aprendizaje y a la participación. Revista Educación Inclusiva, 5 (1), 71-82.

Moriña, A. y Parrilla, A. (2006). Criterios para la formación permanente del profesorado en el marco de la educación inclusiva. Revista de Educación, 339, 517-539.

Muntaner, J. J. Rosselló, M. R. y De la Iglesia, B. (2016). Buenas prácticas en educación inclusiva. Educatio Siglo XXI, 34 (1), 31-50. Disponible en http://dx.doi. org/10.6018/j/252521

Palomares, A. (2011). El modelo docente universitario y el uso de nuevas metodologías en la enseñanza, aprendizaje y evaluación. Revista de Educación, 355, 591-604.

Parajes, M. J. y López, M. (2002). Para poder trabajar por proyectos de investigación en el aula, primero debemos conocernos. Revista Educación Inclusiva, 5 (1), 83-94.

Parrilla, A. (2002). Acerca del origen y sentido de la educación inclusiva. Revista de Educación, 327, 11-29.

Puig, G. y Palos, J. (2006). Rasgos pedagógicos del aprendizaje-servicio. Cuadernos de Pedagogía, 357, 60-63.

Puig, J.M., Batlle, R., Bosch, C. y Palos, J. (2007). Aprendizaje Servicio. Educar para la ciudadanía. Barcelona: Octaedro.

Puig, J. M., Gijón, M., Martín, X. y Rubio, L. (2011). Aprendizaje-servicio y Educación para la Ciudadanía. Revista de Educación, número extraordinario 2011, 45-67.

Zayas, B. y Martínez-Usarralde, M. J. Actitudes del alumnado universitario ante la comunidad: Conciencia de ciutadania y ApS. RIDAS, Revista Iberoamericana de Aprendizaje Servicio, 3, 55-65. 
